\title{
An international expert consensus of management of recurrent hepatocellular carcinoma: a viewpoint
}

\author{
Kuniya Tanaka, Yuki Takahashi, Tetsuji Wakabayashi \\ Department of Gastroenterological and General Surgery, Showa University Fujigaoka Hospital, Yokohama, Japan \\ Correspondence to: Kuniya Tanaka. Department of Gastroenterological and General Surgery, Showa University Fujigaoka Hospital, Yokohama, Japan. \\ Email: u1792ts235@med.showa-u.ac.jp. \\ Provenance and Peer Review: This article was commissioned by the editorial office, Hepatobiliary Surgery and Nutrition. The article did not undergo \\ external peer review. \\ Comment on: Wen T, Jin C, Facciorusso A, et al. Multidisciplinary management of recurrent and metastatic hepatocellular carcinoma after resection: \\ an international expert consensus. Hepatobiliary Surg Nutr 2018;7:353-71.
}

Submitted Oct 13, 2019. Accepted for publication Oct 24, 2019.

doi: 10.21037/hbsn.2019.10.23

View this article at: http://dx.doi.org/10.21037/hbsn.2019.10.23

We read with great interest the article titled "Multidisciplinary management of recurrent and metastatic hepatocellular carcinoma after resection: an international expert consensus" (1) published in HepatoBiliary Surgery and Nutrition. Recurrence can follow all treatments, representing a major challenge in treating hepatocellular carcinoma (HCC), as well as the main cause of death following surgery. Presently, guidelines and recommendations for primary HCC are clearly laid out, but recurrent HCC (RHCC) guidelines remain to be established-even though $70 \%$ of patients have recurrence within 5 years (2). This highlights the need for practical, stratified guidelines for continued management of these patients.

The multidisciplinary team members of the West China Hospital developed a consensus concerning complete management of RHCC, based on their experience and previously published data. A draft consensus written by this multidisciplinary team was refined by experts from both elsewhere in China and other countries. This consensus report includes 10 recommendations and a decision-making path. For each recommendation, supporting evidence is graded at 1 of 6 levels and strength of recommendation at 1 of 5 ranks.

The 10 recommendations concern management of patients at risk of recurrence after primary HCC resection; follow-up after first hepatectomy; adjuvant therapy after first hepatectomy according to risk of recurrence; origins of intrahepatic RHCC; and mechanisms underlying RHCC (intrahepatic metastasis vs. multicentric occurrence).
Further, value of repeat resection and liver transplantation for intrahepatic RHCC, benefit from ablation, indications for transarterial chemoembolization (TACE), and indications for radiotherapy to intra- and extrahepatic RHCC are considered.

In addition to these recommendations, diagnostic criteria including history of hepatitis; imaging features as specified by the Liver Imaging Reporting and Data System (LI-RADS) that are useful in assessment of RHCC; earlier RHCC detection using GALAD and BALAD scores; risk factors predicting RHCC after primary resection; hepatectomy procedures that lessen recurrence; and adjuvant therapies including antiviral agents, molecular targeted therapy, systemic chemotherapy, and immunotherapy are described.

Until now, evidence supporting most therapeutic options for RHCC has been limited to cohort investigations. Randomized controlled studies have been few. Treatments have not been compared in terms of use at specific tumor stages. As a result, therapies commonly used for primary tumors including surgical resection, liver transplantation, TACE, local ablative therapy, and radiotherapy have been applied empirically to recurrences. In contrast to RHCC, many treatment algorithms and treatment assignment schemes based on tumor stage exist for primary HCC, such as the Barcelona Clinical Liver Cancer staging system $(3,4)$, the Japan Society of Hepatology (JSH)-Liver Cancer Study Group of Japan consensus-based treatment algorithm (5), the JSH-HCC guidelines (6), and others. 
The decision-making path for RHCC proposed in the article follows the form of previously proposed algorithms or strategies for primary HCC. Treatment options are narrowed according to successive decision factors regarding host or tumor attributes.

Differences between this RHCC algorithm and those used for primary HCC include a decision path based on recurrence risk factors and characteristics of the primary HCC but not vascular invasion by the RHCC. Risk factors for new recurrences include an interval from resection to recurrence of under 1 year, presence of vascular invasion in the resected primary HCC, and multiple tumors at the time of primary resection. The reason for the change may be low frequency of major vascular invasion detected by imaging at recurrence because of prior detailed imaging following the first hepatectomy. When a portal vein tumor thrombus (PVTT) is recognized, the treatment path recommended by the Chinese Expert Consensus regarding HCC with PVTT depends on extent of tumor thrombus (7).

Another difference concerns molecular-targeted therapy. Based on the SHARP and REFLECT studies, molecular-targeted therapies using sorafenib or lenvatinib are included as options in algorithms for primary HCC. Apparently because of lack of evidence, monoclonal antibody treatments are included in the RHCC algorithms only as a complementary option. Instead, radiotherapy is recommended when extrahepatic metastasis coexists with RHCC, according to the $10^{\text {th }}$ recommendation.

Salvage liver transplantation permits removal of tumors with the widest possible margin together with intrahepatic metastases, while replacing cirrhotic liver parenchyma that would predispose to both hepatic decompensation and multicentric carcinogenesis. The $8^{\text {th }}$ consensus recommendation for RHCC states that salvage transplantation can be performed if a patient's tumor stage falls within the specific enlistment criteria followed by various liver transplantation centers; in contrast, transplantation for primary HCC generally is limited to patients meeting the Milan criteria or having with ChildPugh $\mathrm{C}$ background liver cirrhosis.

The decision-making path for RHCC is well organized overall. However, just as the $4^{\text {th }}$ set of JSH guidelines added vascular invasion to other decision factors in their treatment algorithm (6), vascular invasion by recurrent tumor may become a decision factor in RHCC algorithms. Factors such as adhesions from previous operations and tumor located near major hepatic vessels or bile ducts in the liver remnant may preclude further resections or salvage transplantation, requiring alternative treatments. Accordingly, operative procedure and extent of resection at first hepatectomy should be considered in the decision-making path. Further, as the authors described in their $5^{\text {th }}$ recommendation, recurrent intrahepatic HCC may show a pattern representing either multicentric origin, with better outcome and lower risk of death, or, more ominously, intrahepatic metastasis following treatment. Discriminating between these 2 recurrence mechanisms is crucial in treatment decisions. Number of tumors and background liver status, identified as factors in decision-making for RHCC, may be indirectly related to mechanism of recurrence. However, characterizing genetic differences may be more helpful in distinguishing the 2 mechanisms, as the authors described. RHCC algorithms should become more precise after incorporating these differences in recurrence mechanism.

External validation of this decision-making path in cohorts of patients with different baseline demographics and clinical features will be required. However, we believe that the recommendations and decision-making path will prove helpful in management of RHCC worldwide. As the authors described, ongoing updates and improvements will increase the value of this approach.

\section{Acknowledgments}

Funding: None.

\section{Footnote}

Conflicts of Interest: All authors have completed the ICMJE uniform disclosure form (available at http://dx.doi. org/10.21037/hbsn.2019.10.23). The authors have no conflicts of interest to declare.

Ethical Statement: The authors are accountable for all aspects of the work in ensuring that questions related to the accuracy or integrity of any part of the work are appropriately investigated and resolved.

Open Access Statement: This is an Open Access article distributed in accordance with the Creative Commons Attribution-NonCommercial-NoDerivs 4.0 International License (CC BY-NC-ND 4.0), which permits the noncommercial replication and distribution of the article with the strict proviso that no changes or edits are made and the original work is properly cited (including links to both the formal publication through the relevant DOI and the 
license). See: https://creativecommons.org/licenses/by-nc$\mathrm{nd} / 4.0 /$.

\section{References}

1. Wen T, Jin C, Facciorusso A, et al. Multidisciplinary management of recurrent and metastatic hepatocellular carcinoma after resection: an international expert consensus. Hepatobiliary Surg Nutr 2018;7:353-71.

2. Llovet JM, Burroughs A, Bruix J. Hepatocellular carcinoma. Lancet 2003;362:1907-17.

3. Llovet JM, Brú C, Bruix J. Prognosis of hepatocellular carcinoma: the BCLC staging classification. Semin Liver Dis 1999;19:329-38.

4. Bruix J, Sherman M, Practice Guidelines Committee, et

Cite this article as: Tanaka K, Takahashi Y, Wakabayashi T. An international expert consensus of management of recurrent hepatocellular carcinoma: a viewpoint. HepatoBiliary Surg Nutr 2020;9(3):394-396. doi: 10.21037/hbsn.2019.10.23 al. Management of hepatocellular carcinoma. Hepatology 2005;42:1208-36.

5. Kudo M, Matsui O, Izumi N, et al. JSH consensusbased clinical practice guidelines for the management of hepatocellular carcinoma: 2014 update by the liver cancer study group of Japan. Liver Cancer 2014;3:458-68.

6. Kokudo N, Takemura N, Hasegawa K, et al. Clinical practice guidelines for hepatocellular carcinoma: the Japan society of hepatology 2017 (4th JSH-HCC guidelines) 2019 update. Hepatol Res 2019;49:1109-13.

7. Cheng S, Chen M, Cai J, et al. Chinese expert consensus on multidisciplinary diagnosis and treatment of hepatocellular carcinoma with portal vein tumor thrombus: 2016 edition. Oncotarget 2017;8:8867-76. 